\title{
Production of Bioethanol from Tropical Woody Biomass
}

\author{
Abu Saleh Ahmed ${ }^{1}$, Md. Saiful Islam²*, Md. Ashraful Hoque ${ }^{2,3}$ and \\ Yogeswaran Doraisingam ${ }^{1}$ \\ ${ }^{1}$ Department Mechanical and Manufacturing Engineering, Universiti Malaysia Sarawak 94300 Kota Samarahan, Sarawak, \\ Malaysia. \\ ${ }^{2}$ Department of Chemistry, Faculty of Science, Universiti Putra Malaysia, Selangor, Malaysia. \\ ${ }^{3}$ Department of Biochemistry and Molecular Biology, Faculty of Science, University of Rajshahi, Rajshahi-6205, Bangladesh. \\ *Corresponding author: msaifuli2007@gmail.com
}

\begin{abstract}
:
In this study, we used the hydrolysis fermentation method to convert woody biomass, corn stove, sawdust and oil palm empty fruit bunch (OPEFB) to bioethanol. The samples were first pretreated and hydrolyzed to obtain sugar. The sugar content were measured and recorded. Upon the addition of the yeast peptone dextrose (YPD) medium, the biomass solutions were fermented using conventional yeast and the ethanol obtained were measured and analyzed. The result shows that the OPEFB has the highest sugar content followed by the corn stove waste and finally the sawdust waste. The amount of ethanol obtained in the fermentation process was in line with the amount of mixed sugar content in the hydrolyzed solutions. The OPEFB exhibits the highest ethanol yield, followed by the corn stove waste, and finally the sawdust waste.
\end{abstract}

Keywords:

Woody Biomass; Hydrolysis Fermentation; Bioethanol

\section{INTRODUCTION}

Global warming is of everyone's concern nowadays. Depleting fossil resources due to excessive use of fossil fuels is also a worrying issue needed to be addressed. With so many energy issues and controversies in the world, biomass becomes an attractive and serious topic for many. There are many definitions for biomass. Biomass can be defined as a renewable energy source, biological material derived from living or recently living organisms $\underline{[1,2]}$. Biomass is usually a plant matter grown to produce heat or generate electricity [3]. In order to promote biodiversity and sustainable and healthy forest and ecosystem management and to meet local and regional bioenergy needs, woody biomass will be a critical part of biomass supply mix in the future bio-economy [4-6]. Biomass for energy can include a wide range of materials. High value material for which there is an alternative market, such as good quality, large timber, are very unlikely to become available for energy applications. However there are huge resources of co-products, residues and waste that exist. Such wastes could potentially become available in quantity at relatively low cost or even negative cost where there is currently a requirement to pay for disposal. There are five basic categories of material which could be mentioned. The first would be the virgin wood 